\title{
Educación rural en América Latina: escenarios, tendencias y horizontes de investigación
}

\section{Rural Education in Latin America: scenarios, trends and research horizons}

\author{
Lucila Galván Mora*
}

Recibido: 17 de abril de 2020 Aceptado: 3 de junio de 2020 Publicado: 31 de julio de 2020

To cite this article: Galván, L. (2020). Educación rural en América Latina: escenarios, tendencias y horizontes de investigación. Márgenes Revista de Educación de la Universidad de Málaga. 1 (2), 48-69

DOI: https://doi.org/10.24310/mgnmar.v1i2.8598

\section{RESUMEN}

Este artículo aborda temas relacionados con las políticas y tendencias de investigación de la educación rural en tres países latinoamericanos: México, Colombia y Perú. Los hilos conductores son el interés renovado que existe en la región por el conocimiento de la escuela rural, el diálogo establecido -no libre de tensiones-con las políticas educativas y la búsqueda compartida de una escolaridad inclusiva en los territorios rurales.

El relato destaca rasgos de los contextos particulares donde se produce conocimiento y se dirime el futuro de la escuela rural; reseña tendencias y temas de investigación que germinan en el paisaje; y refiere algunas acciones públicas que, en cada país, se están emprendiendo para la mejora. A manera de cierre, se plantean reflexiones sobre los principales rumbos del trabajo investigativo en la región y los horizontes que se vislumbran para la educación de la población rural.

Palabras clave: educación rural; políticas; investigación educativa; temas emergentes

\section{ABSTRACT}

This article addresses issues related to research policies and trends in rural education in three Latin American countries: Mexico, Colombia and Peru. The guiding threads are the renewed interest that exists in the region for knowledge of rural schools, the established dialogue -not free of tension- whit educational policies and the shared search for inclusive schooling in rural areas.

The story highlights features of the particular contexts where knowledge is produced and the future of the rural school is settled; it reviews trends and research topics that germinate in the landscape; and it refers to some public actions that, in each country, are being undertaken for improvement. By way of closing, reflections are made on the main directions of research work in the region and the horizons looming for the education of the rural population.

Keywords: rural education; policies; educational research; emerging issues 


\section{INTRODUCCIÓN}

La educación rural ha sido un campo soslayado, casi olvidado, por la investigación educativa desarrollada en la mayoría de países latinoamericanos. Las razones de esta dejación, identificadas en un reciente estado del arte realizado en México (Rebolledo y Torres, 2019), parecen estar vinculadas a tres orientaciones en la producción de conocimiento sobre el campo.

La primera razón obedece a la mirada urbano-céntrica que predomina en algunos acercamientos analíticos (Santos, 2019). Esta mirada se nutre de modelos educativos generados para contextos urbanos y oscurece las condiciones de la escolaridad en el medio rural, instalando además una visión deficitaria de la educación rural que ha dificultado seriamente el análisis de su problemática y su especificidad.

La segunda remite a la centralidad que la educación indígena ha tenido en la investigación, postergando el estudio de procesos educativos que ocurren en escuelas de variados contextos rurales, con población diversa. Desde esta postura, la escuela rural no indígena resulta invisible o de escasa importancia para los afanes investigativos. La postergación de su estudio ha derivado en un vacío de conocimiento que es necesario saldar.

La tercera razón tiene que ver con la proliferación de estudios historiográficos sobre educación rural, en detrimento del análisis de su problemática actual. La vasta producción y los aportes de esta línea de investigación, relevantes sin duda, han opacado los incipientes esfuerzos para conocer el sentido de la escuela en los territorios rurales contemporáneos (Torres, 2019).

En este panorama, en medio de olvidos o descuidos involuntarios, en la región se aprecia un creciente interés por el estudio metódico de la educación rural en diferentes contextos culturales. Despuntan vetas inexploradas, nuevos objetos de estudio y temas emergentes en el campo. El trabajo de investigación retoma pasos perdidos y se intenta subsanar la posible indiferencia con estudios puntuales ${ }^{1}$. La escuela rural aflora de ese mar anónimo al que parecía estar destinada.

Este artículo trata precisamente de ese interés renovado por la educación rural, que advierto en tres países latinoamericanos: México, Colombia y Perú. Presento cada caso como un todo integrado. Primero, apunto rasgos del contexto particular donde se construye conocimiento y se dirime el futuro de la escuela rural. Enseguida, reseño temas emergentes y tendencias en la

1 Véase por ejemplo: Rodríguez (2018); Cano e Ibarra (2018); Cardona (2018); Juárez (2016, 2020); Ames (2017); Arias (2017); Zamora y Mendoza (2018). 


\section{ESTUDIOS Y ENSAYOS}

investigación que aprecio en el paisaje. En tercer término, señalo las principales acciones que, a mi modo de ver, se están emprendiendo para atender la educación rural, en cada país. A manera de cierre, comparto algunas reflexiones sobre los horizontes de investigación que se vislumbra en la región ${ }^{2}$.

Las fuentes de información utilizadas para este relato son diversas. Por supuesto, realicé una revisión de la literatura reciente sobre el tema en cada país. Libros y revistas especializadas fueron fuentes privilegiadas para identificar intereses y rumbos de la investigación. Conferencias, notas periodísticas, convocatorias, decretos, leyes, entre otros documentos, aportaron valiosos datos para advertir el carácter contextual de la producción de conocimiento.

Así mismo, los Coloquios Iberoamericanos de Educación Rural que la Red Temática de Investigación de la Educación Rural (RIER) ha organizado en cada uno de los países mencionados, constituyeron excelentes espacios académicos para registrar ideas, preocupaciones y debates importantes que nutren este artículo ${ }^{3}$. En estos espacios, cargados de vida y rebeldía, confluyeron numerosos investigadores y circularon búsquedas compartidas sobre el sentido de la escuela rural contemporánea. Espero trasmitir la aspiración colectiva, manifiesta en cada foro, de incidir en las políticas públicas de nuestros países.

\section{VISIBILIZAR LA ESCUELA MULTIGRADO Y SU POTENCIAL PEDAGÓGICO. EL CASO DE MÉXICO}

\subsection{Rasgos contextuales}

Actualmente, en México está en marcha una política de concentración de niños, niñas y jóvenes de localidades rurales dispersas, en grandes centros escolares ubicados en zonas urbanizadas, con el consecuente desarraigo de sus lugares de origen, la separación de sus familias y el cierre de las “pequeñas” escuelas comunitarias que, por lo general, son de organización multigrado y unitarias, atendidas por un único docente.

La llamada política de "consolidación de servicios educativos" ${ }^{4}$ inspirada en premisas de la OCDE, coloca la relación costo-beneficio como parámetro principal de calidad educativa

2 Agradezco las aportaciones de Ana María Cadavid Rojas, Lydia Espinosa Gerónimo y Paola Arteaga Martínez, colegas pertenecientes a la Red Temática de Investigación de Educación Rural (RIER); y de Damián Báez Galván, docente de la Benemérita Escuela Normal Veracruzana "Enrique C. Rébsamen".

3 Me refiero a los siguientes coloquios: 1er Coloquio Iberoamericano de Educación Rural. Octubre 2016. CDMX. Universidad Iberoamericana, Santa Fe; 3er Coloquio Iberoamericano de Educación Rural. Noviembre 2018. Cusco, Perú. Universidad Nacional de San Antonio Abad del Cusco; y $4^{\circ}$ Coloquio Iberoamericano de Educación Rural. Octubre 2019. Rionegro, Colombia. Universidad Católica de Oriente.

El $2^{\circ}$ Coloquio Iberoamericano de Educación Rural, se realizó en Octubre del 2017, en Canelones, Uruguay, en el Centro Agustín Ferreiro; pero éste no fue referente en el presente artículo.

4 En México, la política en cuestión ha recibido distintos nombres. Al inicio de su implementación se conoció como Proyecto de concentración de escuelas rurales; posteriormente se llamó Programa de reorganización y consolidación de los servicios de CONAFE. En algunas entidades, incluso ha recibido nombre propio, como en Campeche, donde se asume como el programa local "Aprender en Grande". 


\section{ESTUDIOS Y E N SAYOS}

y pretende optimizar el gasto que implica atender "muchas escuelas con pocos alumnos" en los territorios rurales del país (Juárez, 2018). Una visión mercantil que ha sido ampliamente cuestionada, por ignorar el derecho fundamental de niños y jóvenes a recibir educación en su propia localidad (Booth y Ainscow, 2000; Morales, 2012; Naranjo, 2019), y pasar por alto el "despojo cultural" que sufren las comunidades rurales cuando se clausura "su" escuela (Arzola, 2007; Williamson, 2010; Boix, 2014).

Esta política despuntó en el pasado periodo gubernamental (2012-2018) con acciones piloto desarticuladas, que provocaron una oposición tímida y aislada de las comunidades afectadas. En la presente administración, las acciones cobraron fuerza y se hicieron más sistemáticas, en el marco de un discurso que postula una "Nueva Escuela Mexicana” y atención al rezago educativo en zonas de alta y muy alta marginación.

Así, en marzo del 2019, se inaugura el primer "Centro Integral de Aprendizaje Comunitario" (CIAC), en Piedra Hincada de la Soledad, en el Estado de Puebla, el cual concentra alumnos indígenas de todos los niveles de educación básica, provenientes de localidades relativamente cercanas entre sí (Excélsior, 2019) ${ }^{5}$.

Este centro, a cargo del Consejo Nacional de Fomento Educativo (CONAFE) ${ }^{6}$, se presenta como un modelo innovador para el medio rural. Brinda alimentación y hospedaje para alumnos y docentes; opera con un maestro para cada grado escolar, ofrece clases en ngigua -idioma original de la región- y español, promueve la tutoría entre pares como método pedagógico y fomenta la educación comunitaria (Excélsior, 2019).

Durante el año 2019, se instalaron doscientos CIAC en regiones donde se concentra población indígena, y se proyecta abrir ochocientos centros más en los próximos años, afectandolocalidades con menos de doscientos cincuenta habitantes. Además, se anunciaron ajustes en la estructura institucional del CONAFE para profesionalizar a los líderes educativos, mejorar sus condiciones de trabajo y fortalecer su trabajo docente con un acompañamiento pedagógico efectivo ${ }^{7}$. Las acciones se sostienen con fondos del programa "La Escuela en Nuestra” (SEP, 2019), puesto en marcha en esta coyuntura ${ }^{8}$.

Desde la perspectiva oficial, los CIAC son planteles escolares donde "confluyen dos o más centros comunitarios de diferentes localidades". Conforman la principal estrategia para “concentrar servicios educativos dispersos” (DOF 03/10/2019) que, abandonados por décadas,

5 El CIAC mencionado se inauguró con un total de 199 alumnos: 46 niños en educación inicial, 32 en preescolar; 95 en primaria y 26 en secundaria; que provenían de localidades del municipio de Tlacotepec de Benito Juárez, Puebla (Excélsior, 2019).

6 El CONAFE es un organismo público descentralizado de la Secretaría de Educación Pública (SEP), encargado específicamente de la educación inicial y básica de niños y jóvenes de sectores vulnerables del país; cuyos objetivos son combatir el rezago educativo y generar equidad. Por decreto oficial tiene las atribuciones de desarrollar nuevos modelos educativos, capacitar a las figuras docentes y aplicar acciones compensatorias en zonas rurales e indígenas.

7 P. Arteaga. Información sobre la propuesta de atención a la educación rural (Comunicación personal). 07 de noviembre de 2019.

8 Este programa se aplica a partir de un acuerdo oficial emitido por la Secretaría de Educación Pública el 3 de octubre de 2019. Su propósito es la entrega directa de recursos para el mejoramiento de los planteles escolares -construcción, mantenimiento, equipamiento- a las comunidades escolares que constituyan un Comité Escolar de Administración Participativa (DOF 03/10/19). 


\section{ESTUDIOS Y ENSAYOS}

no cumplen parámetros de calidad y rentabilidad. En el afán de concentrar servicios y recursos, el desarraigo de los alumnos de sus lugares de origen, su desprendimiento del seno familiar en edades tempranas, así como el cierre de las escuelas locales, se asumen como daños colaterales necesarios.

Experiencias similares documentadas por la investigación, efectuadas anteriormente en México y en otros países, demuestran que más temprano que tarde la “consolidación” concluye en deserción escolar y una mayor exclusión de la población rural ${ }^{9}$.

Esta situación ha provocado una oposición abierta de docentes e investigadores, con propuestas de atención alternativa. La RIER -por ejemplo- pronunció una Carta Abierta al Secretario de Educación Pública (RIER, 2016) cuando se inició el proyecto de concentración, expresando su preocupación por la debilidad técnica y política de los fundamentos. Más tarde, elaboró “Propuestas de Atención para la Educación Rural” (Popoca y Arteaga, 2019), con el fin garantizar el derecho de la población rural a la educación y la equidad.

El simposio "Las políticas de cierre y consolidación de escuelas rurales: críticas y propuestas”, celebrado en el marco del XV Congreso Nacional de Investigación Educativa (COMIE), en noviembre del 2019, constituyó otro evento público. En este espacio, algunas investigadoras de la RIER, con talante crítico, cuestionaron la esencia colonizadora y discriminadora de la política de concentración, los efectos perversos que conlleva y la ambigua legislación que la cobija ${ }^{10}$.

En este clima contestatario, el trabajo investigativo toma un nuevo impulso y la pesquisa enfoca especialmente a las escuelas multigrado, dado que constituyen la opción educativa de más de un millón de niños y jóvenes mexicanos que habitan en territorios rurales ${ }^{11}$.

\subsection{Tendencias en investigación}

En el país coexisten dos vertientes de investigación sobre el tema claramente definidas. Una orientada al análisis de la situación de las escuelas multigrado, la complejidad del trabajo docente y la problemática curricular. Otra, encargada de la evaluación de la política educativa dirigida a la población rural, con un peritaje nunca antes visto sobre las condiciones de operación, atención y recursos destinados a las escuelas multigrado. Ambas vertientes apuntan a visibilizar la escuela rural, colocarla en la agenda pública y distinguir su especificidad.

En la primera vertiente destaca una línea de investigación etnográfica que analiza prácticas y saberes que circulan en aulas multigrado, con notables hallazgos sobre el conocimiento

9 Para un panorama de los efectos de las políticas de cierre de escuelas rurales implementadas en varios países de Iberoamérica, véase a Juárez (2020).

10 Las investigadoras participantes en este simposio fueron Elsie Rockwell, Carmen Núñez y Lucila Galván. El evento fue coordinado por Diego Juárez, responsable técnico de la RIER.

11 Un estudio reciente elaborado por el INEE (Schmelkes y Águila, 2019: 41) reporta que en el ciclo 2016-2017 operaban 52,452 escuelas multigrado de preescolar y primaria general e indígena, telesecundaria y secundaria para migrantes, dependientes de la Secretaría de Educación Pública (SEP); atendidas por 62,781 docentes y con 1,530,374 de alumnos. 


\section{ESTUDIOS Y E N SAYOS}

profesional cotidiano que los docentes construyen en contextos rurales ${ }^{12}$. Algunos estudios en escuelas primarias, han documentado los ajustes y adaptaciones curriculares que concretan los docentes para responder a las plurales necesidades de los alumnos, y ponen en evidencia los procesos reflexivos que implica la delicada tarea (Mercado, 2002; Arteaga, 2011). Otros, muestran las múltiples resoluciones -curriculares, administrativas y organizativas- que conciben docentes de telesecundaria para eludir situaciones que obstaculizan la escolaridad de sus jóvenes alumnos (Sixto, 2014).

Despuntan también interesantes trabajos que, desde una perspectiva sociocultural, abordan los procesos de co-construcción del conocimiento científico en grupos multigrado ${ }^{13}$. De manera rigurosa, indagan sobre la reconfiguración del currículo oficial, en este caso relacionado con la enseñanza de las ciencias, a la luz del conocimiento disciplinar que tienen los docentes ylos saberes locales que poseen los alumnos. Muestran con detalle la naturaleza situada de la enseñanza simultánea, en función de las particularidades de los estudiantes (Arteaga, 2019; Naranjo, 2019).

Estudios como los mencionados actualizaron el debate con la visión del docente como mero ejecutor de un plan prescrito, al mostrar procesos de resignificación del currículo en escuelas rurales. Y se estima que, en los próximos años, aportarán conocimiento suficiente para construir una propuesta educativa multigrado que cubra el vacío curricular hasta ahora existente al interior del Sistema Educativo Nacional (SEN).

Otras aproximaciones cualitativas buscan de manera explicita fomentar la innovación en las escuelas multigrado. Analizan, por ejemplo, las concepciones pedagógicas que subyacen en la práctica docente y la necesidad de hacer "rupturas epistemológicas” para atender la diversidad de los alumnos considerando los avances y las disposiciones de cada uno, no el grado que cursan (Rosas, 2016; 2018). Documentan también la variedad de recursos pedagógicos que circulan en las aulas para favorecer el aprendizaje y la convivencia en grupos con gran diferencia etaria, revelando las posibilidades formativas que brinda su uso diferenciado en el marco de situaciones didácticas compartidas (Galván, Solares y Espinosa, 2018).

En esta misma posición, algunos estudios resaltan la importancia de construir una pedagogía multigrado, colocando en el centro el saber a enseñar y la tríada docente-alumno-saber, para convertir problemas de la enseñanza en oportunidades de innovación. Estos estudios se han concentrado en las asignaturas de matemáticas (Solares y Solares, 2018; Moscoso, Romero y Tejero, 2018) y español (Rodríguez, 2018), promoviendo la participación directa de docentes rurales en la investigación sobre sus propias prácticas. Las vetas abiertas por estos trabajos apuntan a la construcción de una didáctica multigrado para mejorar resultados de aprendizaje en escuelas rurales.

La evaluación de la educación multigrado, como corriente de investigación, se promovió, en su momento, desde el Instituto Nacional de Evaluación Educativa (INEE), a través de un "Proyecto

12 Esta línea se perfiló y consolidó en el Departamento de Investigaciones Educativas (DIE) del CINVESTAV, bajo la dirección de Ruth Mercado y Elsie Rockwell, investigadoras eméritas.

13 Estos estudios se desarrollan en el grupo de investigación formado por Antonia Candela, especialista en enseñanza de la ciencias, en el DIE-CINVESTAV. 


\section{ESTUDIOS Y ENSAYOS}

Nacional de Evaluación y Mejora Educativa de Escuelas Multigrado” (PRONAEME) que se aplicó en varias entidades federativas (INEE, 2017a).

En este marco, escuelas de todos los niveles de educación básica -preescolar, primaria y secundaria- y tipos de servicio que operan en el país -regular, indígena y comunitariofueron objetos de estudio. Se atendió la estadística y conoció la numeralia multigrado a nivel nacional (INEE, 2018). Se identificó la problemática social asociada a la modalidad, como los altos niveles de marginación de las zonas donde se ubican las escuelas, la dispersión geográfica de las comunidades, los movimientos migratorios que implican a los alumnos y la sombra de la inseguridad en ciertas regiones. Se abarcó también la cuestión curricular, confirmando la ausencia de un modelo específico para multigrado y la insuficiencia de materiales adecuados en las aulas (INEE, 2019a).

La información recogida y su análisis fueron insumos para la elaboración de "Directrices para mejorar la educación multigrado” (INEE, 2019b), entendidas como recomendaciones de política pública para el avance progresivo de acciones gubernamentales, con el fin de saldar rezagos acumulados y garantizar la calidad del servicio en comunidades rurales ${ }^{14}$.

La abrogación de la Ley del INEE en mayo de $2019^{15}$, motivó que las directrices emitidas perdieran vigencia como encomiendas de gobierno; no obstante, su publicación puso sobre la mesa el abandono de las escuelas multigrado, así comola urgente necesidad de revisar estrategias y acciones que han resultado ineficaces para su cabal atención.

La evaluación de la política educativa dirigida a territorios rurales, seguramente tendrá continuidad en ámbitos institucionales y académicos. Los estudios recientes en la materia abrieron un filón que promete información valiosa para reorientar la acción pública relacionada con la escolaridad de la población rural y sus derechos inalienables.

\subsection{Acciones públicas}

Algunas acciones oficiales, emprendidas recientemente, apuntan claramente a fortalecer la política de concentración de servicios y cierre de escuelas rurales multigrado. La instalación de CIAC, como ya apunté, se apoya con recursos financieros adscritos al programa "La Escuela es Nuestra", que prevé la asignación de hasta \$500,000.00 (medio millón de pesos mexicanos) al Comité Escolar de Administración Participativa que se conforme en cada uno de ellos ${ }^{16}$. La finalidad explícita de esta erogación es “promover la concentración de servicios educativos dispersos”, eslogan que trata de ocultar la concentración de alumnos y el cierre de escuelas en pequeñas localidades.

14 Otras directrices que se emitieron como producto de las evaluaciones, fueron: Directrices para mejorar la atención educativa de niñas, niños y adolescentes de familias de jornaleros agrícolas migrantes (INEE, 2016) y Directrices para mejorar la atención educativa de niñas, niños y adolescentes indígenas (INEE, 2017b).

15 Ver Decreto que incluye la abrogación de la Ley del Instituto Nacional para la Evaluación de la Educación en Diario Oficial de la Federación (DOF) 15/05/2019.

16 Estos beneficios están destinados, en primer término, a escuelas ubicadas en zonas con alta y muy alta marginación, con alumnado indígena y de organización completa. En segundo, a las escuelas que con igual grado de marginación no tienen alumnos indígenas y son multigrado (SEP, 2019). 


\section{ESTUDIOS Y ENSAYOS}

Para asegurar que los CIAC reciban los beneficios, se acordó calificar a los centros comunitarios incorporados como escuelas de organización completa, aunque no lo fueran en sí mismos (DOF 03/10/2019). En el mismo sentido, el CONAFE cambió sus reglas para que estos centros ampliaran su matrícula a más de 25 alumnos, dado que deben elevarla significativamente -a 151 alumnos y más- para recibir los bonos (Excélsior, 2019).

Se aprueba, además, una nueva Ley General de Educación (DOF 30/09/2019), en la cual, por primera vez en el país, se reconoce que la educación multigrado merece una legislación exprofeso. No obstante, el reconocimiento legal resulta contradictorio y se presta a interpretaciones ambiguas, como relato enseguida.

Por una parte, el artículo 43 de la mencionada ley ordena atender las condiciones administrativas, la infraestructura y seguridad de las escuelas multigrado; la capacitación de los docentes para realizar adecuaciones curriculares y garantizar el máximo logro en los aprendizajes y desarrollo integral de los alumnos; la dotación suficiente de materiales y recursos didácticos; y la adaptación del modelo a las características regionales, culturales y lingüísticas de la población. Cuestiones que -vale señalar-la investigación sobre el tema viene proponiendo desde hace décadas para fortalecer la educación multigrado.

Por otra, los ordenamientos parecen desmoronarse en el Artículo transitorio décimo sexto, que dicta:

Las autoridades educativas, en el ámbito de sus competencias, realizarán las acciones necesarias a efecto de que, la educación multigrado que impartan en términos del artículo 43 de la Ley General de Educación, sea superada de manera gradual (DOF 30/09/2019, p.67).

Las preguntas que quedan en el aire son: ¿Qué significa que la educación multigrado "sea superada”? ¿Realmente se quiere fortalecer a las escuelas multigrado, como dicta el artículo 43 ? No queda claro si advertimos que algunos sinónimos de la palabra "superada" son vencida, eclipsada, derrotada, rebasada.

Al parecer, con este transitorio se está anteponiendo un argumento legal para justificar el cierre de pequeñas escuelas rurales ya emprendido por la presente administración, pese a las múltiples advertencias que la investigación ha hecho sobre los riesgos de una mayor exclusión de la población rural. La llamada política de consolidación tiene ahora un piso legal para expandirse por todo el territorio nacional.

\section{CONSTRUIR LA PAZ DESDE LA ESCUELA. EL CASO DE COLOMBIA}

\subsection{Rasgos contextuales}

En Colombia, una prioridad social es la construcción y consolidación de la paz, después de un largo conflicto armado que dejó huellas imborrables de violencia, horror y miedo. La escuela se concibe como un "territorio de paz", como espacio comunitario privilegiado para reconstruir 


\section{ESTUDIOS Y E N SAYOS}

el tejido social, la convivencia pacífica y la civilidad, sobretodo en regiones rurales fuertemente golpeadas por la tragedia, como el Oriente Antioqueño o el Norte de Santander.

La aspiración de paz y bienestar permea el ambiente y la actividad social. Y el trabajo de investigación no está al margen de este anhelo, al contrario, en la producción investigativa se aprecia la clara intención de rescatar la memoria viva de adversidades pasadas, reconocer a sectores de población olvidados -campesinos, afrodescendientes, desplazados-y defender el territorio.

En este contexto, varios sectores se organizan para plantear demandas y hacer valer su voz. Como digno ejemplo, menciono el caso de un grupo de mujeres del municipio de Alejandría, que se conformó para elaborar duelos colectivos, sanar heridas y trabajar por la paz permanente. La agrupación se llama formalmente "Asociación de Mujeres Víctimas Cabeza de Familia" (AMUVICAFA); pero se reconocen como las Abrazadas, debido a que "entre pasos y abrazos resignificamos nuestras vidas, nuestras familias y la sociedad” (Calderón, 2019).

En el ámbito de la investigación, las aspiraciones colectivas se hicieron patentes en el reciente “Congreso Internacional Epistemologías del Sur y Ruralidades Latinoamericanas”, realizado en octubre del 2019. Las espirales de reflexión abrieron la discusión de temas emergentes derivados de las difíciles situaciones vividas, anticipando caminos y horizontes nuevos, tales como Educación rural, despojo y retorno; Pedagogía de la Madre Tierra y Pedagogía de la resistencia; Ecología de saberes y diálogos interculturales; Soberanías, buen vivir y tecnologías para la vida; Territorio, mujer y ampliación de la ciudadanía; Espiritualidades latinoamericanas, prácticas y saberes ancestrales.

En Colombia, la construcción de conocimiento sobre la educación rural también está atravesada por un movimiento que busca referentes, metodologías y nociones adecuadas para las circunstancias y realidades de los países de la región, el cual pretende "interpelar el cuerpo teórico unificado y eurocéntrico desde donde se ha venido leyendo la ruralidad latinoamericana" (Convocatoria, 2019).

“Epistemologías del Sur” es el nombre que recibe este movimiento, porque plantea una discusión de fondo con referentes y procederes "hegemónicos”, nacidos en latitudes ajenas, distantes de la historia e identidad cultural que distingue a los países del sur del continente.

Una conferencia impartida por Alfredo Ghiso (2019), en el seno del congreso mencionado, me permitió identificar los principales planteamientos de esta perspectiva austral. Comparto aquellos que -a mi modo de ver- están implicados en el quehacer investigativo relacionado con la educación rural. Epistemologías del Sur propone:

1. Una mirada teórica pero también emotiva, mejor dicho emocionada, hacia la educación. De forma tal que la problematización de una situación educativa, siempre sea un proceso “sentipensante”, articulado a la práctica, la experiencia y el sentir. Razón, deseos, emociones y utopías, conforman necesariamente todo objeto de estudio.

2. Una atención puntual al contexto donde suceden y se analizan las situaciones, porque el conocimiento es histórico, situado en tiempo y espacio determinados, permeado por 


\section{ESTUDIOS Y ENSAYOS}

significados culturales. El origen de toda problematización es contingente, una cuestión crucial para derivar acciones consecuentes y anticipar sus resultados.

3. Una concepción del maestro como sujeto político, fundamentalmente como un sujeto político que es perfectamente capaz de la trasformación social. Nada es imposible de cambiar, reza un principio de esta perspectiva epistemológica. El cambio es una opción política y ética. En ese sentido hay una ruptura con la ingenuidad, con la postura que supone neutralidad del conocimiento y sus usos sociales.

4. Una aceptación del conflicto como base de las relaciones sociales y la construcción del conocimiento. En este sentido se afirma el carácter dialógico del conocimiento, la ruptura de certezas, la apertura al riesgo y a la duda existencial. Se trata de mirar desde otros lugares culturales para apropiarse críticamente de la realidad.

5. Un pensamiento estratégico basado en la investigación-acción-participativa, como procedimiento metodológico y ético para construir conocimiento en colectividad, sustentado en la creatividad-imaginación-coraje como actitud vital para cambiar las cosas y fundamentado en la situación-memoria-conciencia como ruta crítica para la participación y la emancipación.

Otro rasgo contextual de la investigación en Colombia es la puesta en marcha de Modelos de Educación Flexible (MEF) en el medio rural, como estrategia para mejorar el logro académico de estudiantes, minimizar los índices de deserción y fortalecer el vínculo con las comunidades. Entre los distintos $\mathrm{MEF}^{17}$, destaca el programa “Escuela Nueva” que, implementado desde la década de los 60, promueve la experimentación pedagógica, la enseñanza multigrado, el autogobierno de los alumnos y la vinculación con organizaciones locales (Rivera y Zavala, 2019).

El impulso que han tenido los MEF y, en especial, la "Escuela Nueva” en el campo colombiano, ha generado una corriente de análisis sobre sus logros, vacíos y posibilidades de mejora, con importantes hallazgos para replantear programa y políticas educativas ${ }^{18}$. La impronta de renovación que la aplicación de los MEF ha dejado en la memoria colectiva, condiciona de alguna forma el marcado interés por adelantar una educación innovadora en el momento actual.

\subsection{Tendencias en investigación}

La investigación en Colombia se distingue por su tendencia altamente propositiva, orientada a solucionar problemas y rezagos, mejorar resultados de aprendizaje e incidir en las decisiones sobre educación y ruralidad. Desde los albores de la presente década, las investigaciones tuvieron el propósito de vincular la escuela rural al trabajo productivo de las comunidades. Vislumbraron

17 Además de la Escuela Nueva, otros modelos de educación flexible escolarizados son los llamados Aceleración del aprendizaje, Telesecundaria y Posprimaria rural. Existen también modelos no escolarizados, como el Sistema de aprendizaje tutorial (SAT), Servicio de Educación Rural (SER) y el modelo Cafam (Zamora y Mendoza, 2018).

18 Para un análisis detallado de los estudios relacionados con el origen, implementación y evaluación del programa Escuela Nueva, véase a Cadavid, Acosta y Runge (2019). 


\section{ESTUDIOS Y E N SAYOS}

una institución escolar “pertinente”, que impulsara “proyectos pedagógicos productivos”, entendidos como diálogos creativos entre el saber escolar y la vida cotidiana de la población (Gil, Vera y Gil, 2012). La idea de construir una escuela volcada hacia el mundo rural, atenta a las tradiciones y a la vez innovadora, permearía los estudios consecutivos sobre el tema y, de manera importante, las acciones públicas que el Ministerio de Educación Nacional (MEN) impulsaría en años futuros.

Actualmente, abre camino una corriente de investigación que podría denominarse "Pedagogía Rural” (Arias, 2017), la cual busca referentes, métodos y herramientas metodológicas para establecer un currículo contextualizado para los sectores rurales y acercar los modos de aprendizaje campesino a los aprendizajes escolares.

A través de sus propuestas cuestiona la política educativa dirigida a campesinos y afrodescendientes, representantes de la ruralidad colombiana. La marginalidad, las secuelas de la guerra, el desempleo, la segregación racial y los ritmos de la ruralidad han marcado la vida de estos grupos, y la educación que reciben es ajena a estas condiciones, además de precaria. En esta circunstancia, la escuela rural de hoy no solo debe enseñar contenidos escolares, sino reconocer los saberes locales relacionados con el trabajo agrícola, el arraigo a la tierra, la sostenibilidad ambiental y la historia comunitaria (Arias, 2017, 56-61).

Desde esta lógica, los estudios parten de las expectativas sociales hacia la escuela, entran a las aulas para identificar saberes y prácticas locales en los procesos escolares y fomentan la interculturalidad. Esto sin dejar de denunciar los niveles de desigualdad y pobreza que sufre la población rural.

La Pedagogía Rural orienta currículos adaptados a los contextos culturales, que contengan saberes campesinos, preserven la identidad y promuevan dinámicas interculturales. Y hacia ello parece encaminarse su pesquisa de investigación en tiempos venideros.

Por otra parte, la formación de educadores para los territorios rurales constituye una temática en pleno desarrollo. El impulso proviene -principalmente- de la "Línea de Investigación en Educación Rural”, de la Universidad Pedagógica Nacional (UPN) con sede en Bogotá. Esta línea sostiene que la docencia rural tiene una especificidad que ha sido desdibujada tanto por la homogeneización del currículo, que supuestamente alivia desigualdades sociales, como por la “subvalorización” de lo campesino en la idiosincrasia nacional (Zamora y Mendoza, 2018). La afirmación se basa en exploraciones previas que permitieron identificar los rasgos contextuales y personales que integran la identidad docente rural, como los fuertes vínculos con la comunidad, la organización multigrado de la enseñanza, el dilema entre permanecer en el medio o migrar, y la creatividad en juego para enfrentar dificultades y carencias en las aulas (Zamora, 2005).

Los estudios incursionan, además, en escenarios escolares multigrado, vinculados sobretodo al programa Escuela Nueva, para conocer la operación de la modalidad y su articulación con la ruralidad. Han encontrado una fuerte tendencia a mantener la graduación establecida dentro de grupos multigrado, cuando éstos requieren otro tipo de organización; la pervivencia de una estructura jerárquica que limita la autonomía curricular de los docentes; y un acompañamiento pedagógico deficiente que no resuelve necesidades de formación durante el ejercicio profesional (Zamora y Mendoza, 2018). 


\section{ESTUDIOS Y ENSAYOS}

La necesidad de una formación especializada de los docentes rurales se hizo evidente y las instituciones formadoras se enfocaron como objeto de estudio. Ahora se sabe que la mayoría de ellas conservan lejanía, física y académica, con los territorios rurales. En el mejor de los casos, los vínculos se reducen a prácticas de los estudiantes de magisterio en escuelas rurales; pero sin atender la necesaria adecuación de los planes de estudio para el trabajo educativo en "esa otra Colombia”, campesina y rural (Zamora y Mendoza, 2018, 76).

Ante esta problemática, la línea de investigación mencionada se propuso, por un lado, redimir el desinterés aparentemente generalizado por la educación rural; y, por otro, incentivar propuestas de formación de educadores para zonas rurales, hasta ahora prácticamente ausentes en el país ${ }^{19}$ (Zamora y Mendoza, 2018).

El tema de la formación profesional de docentes rurales, por su importancia estratégica, ha sido ampliamente estudiado desde una perspectiva de la interculturalidad. Los esfuerzos se enmarcan en programas para la protección de la infancia, como el llamado "Con paz aprendemos todos” - dirigido a docentes afrocolombianos de los departamentos de Chocó y Putumayo-, que buscan integrar la historia y la cultura de las diferentes etnias a la vida escolar, valorar los saberes ancestrales y concretar un currículo con enfoque etnoeducativo (Sánchez, 2018).

Algunos autores afirman que, en relación a este tema, son reiterativos los señalamientos para atender la formación inicial y continua de los docentes, trascender la capacitación instrumental y fomentar el análisis sobre pedagogía y ruralidad. Contribuir a este análisis es una tarea que la investigación actual asume como impostergable (Cadavid, Acosta y Runge, 2019).

En el panorama colombiano, se aprecia además una veta de investigación etnohistórica que remite a la recuperación de la memoria histórica, como eco de un sentir social que así defiende la paz, el bienestar comunitario y el territorio. La veta se perfiló claramente en el "Congreso de Rionegro" convocado en 2019, en la espiral denominada "Territorio: movimientos sociales, experiencias y narrativas”. Si bien aún es incipiente, puede anticiparse que en los próximos años crecerá en importancia, por su vocación a develar las soterradas “voces del silencio”.

\subsection{Acciones públicas}

En Colombia se impulsa ahora mismo un Plan Especial de Educación Rural (PEER), con el fin de proporcionar educación de calidad y con equidad a la población rural. Dicho Plan deriva del “Acuerdo final para la terminación del conflicto y la construcción de una paz estable y duradera” (2016), anunciado en los diálogos para la paz efectuados en La Habana, en agosto del 2016, y firmado en Bogotá en noviembre de ese mismo año.

El primer punto de este Acuerdo, titulado "Hacia un Nuevo Campo Colombiano: Reforma Rural Integral”, colocó a la población rural en el centro del proyecto de desarrollo económico y social del país, y puso sobre la mesa asuntos relacionados con la tenencia de la tierra, la pobreza

19 Recientemente fueron cerradas las dos únicas Licenciaturas en Educación Rural que existían en Colombia, una en la Universidad Católica de Oriente (Antioquia) y otra en el Centro Universitario de Bienestar Rural del Cauca (Zamora y Mendoza, 2018, 80). 


\section{ESTUDIOS Y ENSAYOS}

extrema y las condiciones para el bienestar común. La educación rural se proyectó, desde ese momento, bajo criterios de cobertura universal y gratuidad, calidad a través de modelos educativos flexibles y equidad en oportunidades educativas. Se anticipó también la intención de fortalecer la investigación, la innovación y el desarrollo científico en el campo (Acuerdo, 2016, 22-23).

Este marco estructural que sostiene los programas y las acciones actuales sobre educación rural, con gran significado histórico, determina en gran parte el interés gubernamental por impulsar modelos innovadores en las comunidades rurales -campesinas, indígenas, afrocolombianas-que han padecido marginación y rezago social.

El MEN estableció rutas de acción para hacer efectivo el derecho a la educación de la población rural. Entre ellas destacan la integración de un fondo de financiamiento para la infraestructura escolar; mayores incentivos para los educadores rurales, como vivienda, trasporte o prima salarial, así como facilidades para formación de posgrado; aplicación de MEF como estrategia pedagógica vinculada a los contextos rurales; y formación situada de docentes en ejercicio. Asimismo, las rutas indican atención al analfabetismo, instalación de centros educativos integrados en zonas rurales dispersas y participación abierta de las comunidades en la gestión, implementación y seguimiento de los servicios educativos (MEN, 2015, 23-49).

Cerrar la brecha rural-urbana y vincular la escuela al territorio y al sector productivo son metas de la política educativa para el campo. Los esfuerzos realizados en ese sentido son importantes; no obstante, aún queda por construir una "institucionalización de la educación rural” que trascienda las respuestas coyunturales y contemple las diversas ruralidades presentes en el país (Parra, et al., 2018).

\section{4. ESCUCHAR Y ATENDER A LA NIÑEZ Y LA JUVENTUD OLVIDADAS. EL CASO DE PERÚ}

\subsection{Rasgos contextuales}

En Perú, como en muchos países de Latinoamérica, la educación de la población rural es uno de los grandes desafíos contemporáneos, para la política y la investigación. Los esfuerzos realizados para mejorar la calidad de los servicios, no han derivado en una estrategia de largo aliento que atienda con pertinencia las necesidades y rezagos de este grupo de población. La brecha de calidad educativa entre zonas urbanas y rurales en este país andino es una de las más grandes de la región (Montero, et. al., 2001).

En el ámbito de la investigación educativa, la educación rural se coloca en el centro del debate sobre los derechos fundamentales de la población, en su mayor parte indígena, que habita en aisladas comunidades andinas o amazónicas. Las indagaciones se dirigen a las experiencias educativas de niños y jóvenes adolescentes dentro y fuera del sistema escolar, con el fin de formular proyectos de intervención educativa desde una perspectiva inclusiva, con orientación bilingüe e intercultural (Ames, 2004).

La preocupación que atraviesa el quehacer investigativo, se refiere a establecer un diálogo permanente con la política educativa para incidir en la calidad y equidad de los servicios. Se 


\section{ESTUDIOS Y ENSAYOS}

espera que los proyectos de intervención se sustenten, cada vez más, en estudios sistemáticos sobre la escolaridad en zonas rurales, y que la educación pública se desarrolle con oportunidad y pertinencia.

Precisamente, el "VI Seminario Nacional de Investigación Educativa” que se llevó a cabo en noviembre del 2018, en la ciudad de Cusco, propuso interesantes cuestiones que invitan al diálogo: ¿Qué sabemos de la educación rural? ¿Las escuelas rurales mejoran la calidad de vida de la población? ¿Qué modelos educativos podemos proponer en zonas rurales? ¿Cómo hacer sostenible una política de educación rural? (Convocatoria, 2018).

La desigualdad social y los movimientos migratorios masivos que se verifican en gran parte de la región, también forman parte de las inquietudes investigativas actuales, por significar un componente estructural de alto impacto en los escenarios rurales. La privatización y orientación mercantil de los servicios educativos que se verifica en Perú, y en varios países vecinos, imponen replanteamientos de fondo, teóricos y metodológicos, para abordar el estudio de la escuela en tiempos de una crisis generalizada.

En este contexto, la colaboración para el desarrollo de investigaciones con una perspectiva latinoamericana, que incidan en las políticas públicas de la región, se vislumbra como la vía más apropiada, en el corto plazo, para encontrar soluciones novedosas a una problemática educativa que traspasa fronteras geográficas y políticas (Convocatoria, 2020).

\subsection{Tendencias en investigación}

La producción peruana sobre educación rural e indígena tiene una honda raíz antropológica. Nació vinculada a la vocación etnográfica de la Antropología Norteamericana, interesada en los procesos de socialización y trasmisión de la cultura, y en describir modos culturales diversos de educar a las nuevas generaciones. Así, el interés por la escolaridad parte de un concepto amplio de educación. No se limita a documentar aspectos relacionados con procesos escolares, sino que abarca experiencias educativas que ocurren en diversos ámbitos sociales, en los cuales niños y jóvenes adquieren saberes, códigos y disposiciones para la vida social (Ames, 2017).

En el Perú, los estudios sobre la escuela rural se iniciaron en localidades indígenas, en la década de 1950, cuando el sistema escolar empezó a llegar a las regiones andinas. Este inicio marcó rumbo y definió el interés que hoy encontramos por la educación de los pueblos originarios. La investigación actual sigue concentrada en comunidades andinas, aunque con aires renovados a partir del desarrollo de un enfoque propio de la interculturalidad ${ }^{20}$. También se ha abierto una línea de indagación en comunidades amazónicas, en las que la presencia de la escuela es más reciente, orientada a conocer la adaptabilidad de esta institución a las condiciones y cosmovisión de una población escasamente atendida (Ames, 2017).

En general, las investigaciones reconocen a los niños, niñas y jóvenes como protagonistas de la escena escolar y los colocan en el centro de la pesquisa etnográfica. La atención a la infancia

20 Para un relato detallado sobre los estudios de antropología y educación en el Perú, véase a Ames (2017, pp. 11-24). 


\section{ESTUDIOS Y E N SAYOS}

y la juventud indígenas proviene de esa raíz antropológica ya señalada, pero también obedece a la intencionalidad de repensar las políticas existentes que, desprovistas de una visión étnica, descuidan calidad y equidad educativas para estos sectores.

Un estudio realizado en la Amazonía peruana explora, por ejemplo, las prácticas y saberes comunitarios que niños shipibo llevan al espacio escolar y cómo intervienen en la organización de su propio aprendizaje. Estos alumnos, como actores interculturales, gestionan vínculos con espacios extraescolares donde trascurre su vida y su actividad: la casa, el río, el bosque y la chacra, para dar sentido al espacio escolar y apropiarse de la escuela (Zegarra, 2017). El análisis muestra cómo el modelo de Educación Intercultural Bilingüe es reconfigurado por los alumnos y sus profesores, construyendo así una "nueva interculturalidad” que el discurso estatal no legitima.

Otro trabajo analiza continuidades y rupturas en las trayectorias escolares de jóvenes amazónicos asháninkas que cursan la educación secundaria. Describe las tensiones entre las dinámicas juveniles propias del entorno cultural y las exigencias escolares impuestas por un sistema ajeno a las prácticas locales (Gastelú, 2017).

El estudio muestra la condición de adultez e independencia que se exige a los jóvenes en los ámbitos comunitarios, y la idea de estudiante sumiso que prevalece en la escuela, como representaciones de mundos aparentemente opuestos. En esta situación, la escuela se convierte en un "espacio de frontera" donde los jóvenes asháninkas afrontan activamente las tensiones y negocian su escolaridad sin perder su identidad (Gastelú, 2017).

En la región andina de Apurímac se realizan interesantes investigaciones sobre el recorrido que mujeres jóvenes, provenientes de familias rurales hablantes de quechua, emprenden para acceder a la educación superior una vez terminado el secundario. Un recorrido plagado de resistencia, desaprobación y eventual desconfianza de parte de padres y hermanos -por no entender la aspiración de las jóvenes a la universidad-y que pone a prueba la agencia de las jóvenes para defender su autonomía y labrar su futuro educativo (Villegas, 2017).

Estas investigaciones muestran que la transición exitosa de algunas jóvenes depende de cierta apertura familiar, apoyos de mujeres cercanas que vivieron experiencias similares y, sobre todo, de "negociaciones interdependientes" donde se juegan sus ambiciones profesionales. Por lo general, ellas acaban alternando los estudios con trabajo remunerado, para pagar la matrícula y los enseres escolares, a cambio del consentimiento familiar. El sistema escolar, indiferente, no prevé estrategia alguna para apoyar el acceso y la permanencia de estas jóvenes en las aulas universitarias (Villegas, 2017).

Estudios como los mencionados son representativos de las tendencias de la investigación actual sobre educación rural. La pesquisa etnográfica se concentra en la escolaridad de los niños y jóvenes indígenas que habitan comunidades andinas y amazónicas, con el fin de escuchar su voz, registrar su testimonio y comprender sus necesidades y expectativas educativas.

La indagación aborda los procesos interculturales que se conforman en escuelas primarias y secundarias, especialmente. $\mathrm{Y}$ se incursiona en las negociaciones que jóvenes indígenas establecen para acceder a estudios universitarios, con una interesante perspectiva de género que 
seguramente moverá estereotipos. El aporte principal de estos trabajos es pensar la educación desde una perspectiva antropológica, considerando la rica diversidad cultural del Perú.

\subsection{Acciones públicas}

En la actualidad, la investigación de la educación rural es un quehacer comprometido con sectores de población que no han sido escuchados por las instancias gubernamentales, y conlleva la clara intención de influir para modificar esta actitud política. Menciono tres acciones que ejemplifican el talante propositivo que impera en los círculos académicos donde se gesta conocimiento e impulsan proyectos.

En primer término están los "Seminarios de Antropología de la Educación”, adscritos a la Sociedad de Investigación Educativa Peruana (SIEP), que se llevan a cabo cada dos años con la finalidad de difundir teorías y evidencias que contribuyan a mejorar la escolaridad en los territorios rurales indígenas. En estos Seminarios se abren debates, establecen alianzas y proponen estrategias para fortalecer la educación rural (Convocatoria, 2018).

En segundo lugar, se encuentra el "Programa de apoyo a jóvenes investigadores que residen en el Perú”, a partir del cual se otorgan becas para incentivar el interés por el trabajo investigativo. La secundaria y los adolescentes constituyen, en este momento, el núcleo al que se dirigen los esfuerzos financieros. Incluso, de manera complementaria, se premian tesis de licenciatura y maestría que atienden temas relacionados con educación secundaria, en respuesta al abandono que ha tenido este nivel (Convocatoria, 2018).

En tercero, se ubican los vínculos entre instituciones y redes de investigación que buscan incidir en las políticas educativas con escala iberoamericana, entre las que destacan la World Education Research Association (WERA), la Red Iberoamericana de Educación en Territorios Rurales (RIBETER) y la misma RIER. Las alianzas han fructificado en coloquios internacionales donde se reúnen investigadores, docentes, estudiantes, funcionarios, y personas interesadas en la educación rural de la región, para el intercambio teórico, la difusión de hallazgos y el impulso de proyectos conjuntos para atender problemáticas educativas compartidas.

\section{5. ¿HACIA DÓNDE VA LA INVESTIGACIÓN DE LA EDUCACIÓN RURAL EN LATINOAMÉRICA?}

Este interrogante fue guía en el recorrido que emprendí por tres países latinoamericanos para conocer escenarios y tendencias de la investigación en educación rural, y me parece pertinente volver a él para ordenar los planteamientos finales. Más que una respuesta concluyente, comparto algunas reflexiones sobre los horizontes que vislumbro para el trabajo investigativo en la región.

Ciertamente, en cada uno de los países tratados, se advierte un interés renovado por el estudio sistemático de la escuela rural y su significado en diversos contextos culturales. El campo de investigación en la región se abre a temáticas que no habían sido abordadas con la profundidad que requieren para conocer la especificidad de la educación rural, concernientes -por ejemploa los saberes docentes que circulan en las aulas, la reingeniería del currículo en los contextos 


\section{ESTUDIOS Y ENSAYOS}

locales, la presencia de la memoria histórica en la vida escolar, la vinculación de los aprendizajes con prácticas y saberes comunitarios o las negociaciones que emprenden niños, niñas y jóvenes para acceder a la escolaridad.

El aire de renovación abarca ámbitos que habían permanecido en la sombra. Los esfuerzos investigativos se centran en modalidades educativas que parecían invisibles, a pesar de su amplia cobertura en zonas rurales, como la escuela multigrado y la telesecundaria en México; en sectores de población olvidados por los gobiernos y la sociedad, como los afrodescendientes o los desplazados por conflictos armados en Colombia; y en comunidades remotas donde la escolaridad es un suceso reciente, como las que habitan la selva amazónica del Perú.

El quehacer investigativo se diversifica notablemente, se formulan nuevas preguntas y se siguen procedimientos metodológicos que dejan a un lado la vieja rencilla "cuanti-cuali", para comprender situaciones educativas desde una perspectiva holística, interdisciplinaria. La dimensión política de la investigación se hace explícita, tanto en el afán de incidir en las políticas educativas de la región, como en la producción de conocimiento para la trasformación social. La búsqueda de una epistemología propia para entender mejor nuestras realidades está en el centro de la labor.

En el panorama latinoamericano se advierten debates centrales sobre la escuela rural y su margen de posibilidad en la sociedad contemporánea. Uno de ellos, referido a la vinculación de la escuela, la actividad productiva y la sinergia de los territorios, exige revisar concepciones estrechas sobre la contextualidad que han impedido trascender el ámbito local. El énfasis que la investigación ha puesto en los contextos de escolaridad ha derivado, en muchas ocasiones, en una adaptación simplificada del currículo a los ámbitos comunitarios, olvidando la necesidad de un desarrollo educativo con principios de universalidad. La escuela rural no puede quedar confinada en su contexto inmediato.

Otro debate se ubica en el afán propositivo de la investigación, en las ideas predominantes sobre la mejora educativa. En ocasiones las propuestas se elaboran con base en la dicotomía rural/urbano, teniendo como referente modelos de acción urbanizados. Se impone, entonces, continuar trabajando en una definición de la ruralidad sin esquemas previos, con mente abierta para abarcar la heterogeneidad del mundo rural. ¿Qué escuelas, qué alternativas pedagógicas? ¿Para qué ruralidades?, podrían ser las preguntas que guiaran la indagación. Pluralidad, la noción nuclear para pensar la mejora, la continuidad y los cambios en educación rural.

Está pendiente, además, la discusión sobre el papel de la escuela en la crisis civilizatoria que ya anticipaba Amin Maalouf (2019) en su reciente libro, y que la pandemia que ahora enfrentamos pone en evidencia. Un naufragio del mundo occidental provocado por la fragmentación, la soberbia identitaria, la legitimación de las disparidades, el extravío ético, el desencanto y la acritud, entre otros males (Maalouf, 2019, 205-267). En esta coyuntura, la investigación tendrá que avanzar con una perspectiva global y francamente humanista. La escuela rural tiene que ser colocada en la vorágine mundial para ubicar su verdadera dimensión, para que pueda contribuir, desde su raíz local, a la conciencia planetaria que supone el vivir juntos y pertenecer a la misma colectividad. 


\section{EST U D O S Y E S A Y S}

Termino el artículo en medio de la constricción impuesta por la catástrofe sanitaria que azota al mundo. En el punto final, con ese sentimiento de irrealidad que nos envuelve a todos, me pregunto qué pasará con la educación rural en los países latinoamericanos, donde la recesión económica -la otra pandemia- que se aproxima inexorablemente ahondará las brechas de la desigualdad. ¿Será la educación de la población rural una prioridad de los gobiernos en el mundo venidero? ¿Alcanzará la imaginación para plantear alternativas a la altura del desastre?

La educación en línea se plantea como principal estrategia ante la emergencia, y los aparatos educativos en nuestros países se aprestan para desarrollarla. En algunos casos, como en México, incluso se han planteado acciones para superar en el corto plazo los enormes rezagos existentes en materia de tecnología y conectividad (MEJOREDU, 2020). Pero, como vemos, la estrategia virtual no necesariamente preserva el derecho a la educación de la población rural; al contrario, parece excluirla con mayor dureza. Tampoco abona a la equidad. Seguramente, pronto tendremos que concebir otras maneras de educar, construir otras escuelas, inéditas.

Hace pocos días leí un mensaje de las ancianas de la Tierra; dicen que los tiempos de penumbra también iluminan, que saldremos de esta experiencia colectiva más sabios, más sabias, con ojos abiertos y espíritu perspicaz. Quizá entonces, con otras luces, podamos reinventar las escuelas rurales, reconociendo su raigón histórico y su aliento futuro.

En los países tratados, la investigación continuará labrando el campo de la educación rural por las sendas trazadas, con perspectivas más amplias y agudas. Con lentitud, como viene el futuro, pero con el optimismo y la "esperanza dura” que Benedetti advirtió en los coterráneos del Sur.

\section{REFERENCIAS}

Acuerdo final para la terminación del conflicto y la construcción de una paz estable y duradera (2016). Recuperado de http://peacemaker.un.org/sites/peacemaker.un.org/files/Colombia\%20Nuevo\%20 Acuerdo\%20Final\%2024\%20Nov\%202016_0.pdf

Ames, P. (Ed.) (2017). La diversidad en la escuela. Aproximaciones antropológicas a las experiencias educativas de los niños, niñas y jóvenes peruanos. Perú: Pontificia Universidad Católica del Perú-Instituto de Investigación para el Desarrollo.

Ames, P. (2004). Experiencias relevantes de educación orientadas al desarrollo rural alternativo en Perú. La nueva ruralidad: Desafíos y propuestas. Perú: Ayuda en Acción.

Arias, J. (2017). Problemas y retos de la educación rural colombiana. Educación y Ciudad (33), JunioDiciembre, 53-62.

Arteaga, P. (2011). Los saberes docentes de maestros de primaria con grupos multigrado. México: Consejo Mexicano de Investigación Educativa.

Arteaga, P. (2019). Prácticas docentes en la organización del currículo de ciencias en una primaria unitaria. Ponencia presentada en el Congreso internacional Epistemologías del Sur y Ruralidades Latinoamericanas. 9-11 de Octubre. Rionegro, Colombia. pdf

Arzola, D. (2007). Los Centros Regionales de Educación Integral. El dilema de la tradición o la innovación. Memoria electrónica IX. Congreso Nacional de Investigación Educativa. Congreso Nacional 


\section{ESTUDIOS Y ENSAYOS}

de Investigación Educativa. Yucatán, México. Recuperado de http://www.comie.org.mx/congreso/ memoriaelectronica/v09/ponencias/at13/PRE1178820230.pdf

Boix, R. (2014). La escuela rural en la dimensión territorial. Innovación educativa, (24), 89-97.

Booth, T. y Ainscow, M. (2000). Índice de inclusión. Desarrollando el aprendizaje y la participación en las escuelas. Bristol, UK: UNESCO-CSIE.

Cadavid A., Acosta, L. y Runge, A. (2019). La educación primaria rural. De posiciones y perspectivas. Teresina, año 24 (43), 384-415.

Calderón, S. (2019). Memorias en vilo: entre la esperanza y la impunidad. Movete (Movimiento Social por la Vida y la Defensa del Territorio) (5) 7.

Cano, A. e Ibarra, E. (Coords.) (2018). Vulnerabilidad, innovacióny prácticas docentes en escuelas multigrado. México: RIER-UPES- Editora Nómada.

Cardona, P.D. (Coord.) (2018). Educación y equidad en contextos rurales: Debates y propuestas. México: Biblioteca LEMI, Universidad Autónoma de Querétaro.

Convocatoria. (2018). IV Seminario Nacional de Investigación Educativa. Perspectivasy desafíos de la educación rural: diálogos entre la investigación y la política educativa. SIEP-RIER-RIBETER. 8-10 de noviembre de 2018. Cusco, Perú.

Convocatoria. (2019). Congreso Internacional Epistemologías del Sur y Ruralidades Latinoamericanas. 9-11 de Octubre. Rionegro, Colombia. pdf

Convocatoria. (2020). XV Simposio Interamericano de Etnografía y Educación. Etnografías educativas en tiempos de crisis y movilidad. 1-3 de junio de 2020. Lima, Perú.

Diario Oficial de la Federación 15/05/2019. Decreto por el que se reforman, adicionan y derogan diversas disposiciones de los artículos 30, 31 y 73 de la Constitución Política de los Estados Unidos Mexicanos, en materia educativa. Recuperado de https://www.dof.gob.mx/nota_detalle.php?.codigo=5560457\&fec $\underline{\text { ha }=15 / 05 / 2019 \& \text { print }=\text { true }}$

Diario oficial de la Federación 30/09/2019. Ley General de Educación. Recuperado de http://www. diputados.gob.mx/LeyesBiblio/pdf/LGE_300919.pdf

Diario Oficial de la Federación 03/10/2019. Acuerdo por el que se emiten los Lineamientos de Operación del Programa La escuela es Nuestra. Recuperado de https://dof.gob.mx/nota_detalle.php? codigo=557440 $\underline{3 \& f e c h a}=03 / 10 / 2019$

Excélsior. 30/03/2019. Inaugura SEP Centro Integral de Aprendizaje Comunitario en zona indígena. Recuperado de https:/www.excelsior.com.mx/nacional/inaugura-sep-centro-integral-deaprendizaje-comunitario-en-zona-indigena/1304817

Galván, L., Solares, D. y Espinosa, L. (2018). Recursos pedagógicos para la enseñanza: una noción desde el aula multigrado. En Cano, A. e Ibarra, E. (Coords.), Vulnerabilidad, innovación y prácticas docentes en escuelas multigrado (61-86). México: RIER-UPES-Editora Nómada.

Gastelú, R. (2017). Una aproximación a los escenarios de continuidad e interrupción escolar desde las experiencias y dinámicas juveniles asháninkas. En Ames, P. (Ed.). La diversidad en la escuela. Aproximaciones antropológicas a las experiencias educativas de los niños, niñas y jóvenes peruanos (pp. 59-89). Perú: Pontificia Universidad Católica del Perú- Instituto de Investigación para el Desarrollo. 


\section{ESTUDIOS Y ENSAYOS}

Ghiso, A. (2019). Epistemologías del Sur, procesos y metodologías participativas. Conferencia magistral. Congreso internacional Epistemologías del Sur y Ruralidades Latinoamericanas. 9-11 de Octubre. Rionegro, Colombia.

Gil, C.J., Vera, N.Y.y Gil, C.A. (2012). Hacia una escuela productiva: la escuela desde el mundo rural. Colombia: Universidad de Pamplona. (Documento de trabajo). pdf

INEE. (2016). Directrices para mejorar la atención educativa de niñas, niños y adolescentes de familias de jornaleros agrícolas migrantes. México: Instituto Nacional de Evaluación Educativa.

INEE. (2017a). Proyecto Nacional de Evaluación y Mejora Educativa de Escuelas Multigrado (PRONAEME). México: Instituto Nacional de Evaluación Educativa.

INEE. (2017b). Directrices para mejorar la atención educativa de niñas, niñosy adolescentes indígenas. México: Instituto Nacional de Evaluación Educativa.

INEE. (2018). Panorama Educativo de México 2018. Indicadores del Sistema Educativo Nacional Educación Básica y Media Superior. México: Instituto Nacional de Evaluación Educativa.

INNE. (2019a) Evaluación integral de la Política Pública de Educación Multigrado. Informe final. México: Instituto Nacional de Evaluación Educativa.

INEE. (2019b). Directrices para mejorar la educación multigrado. México: Instituto Nacional de Evaluación Educativa.

Juárez, D. (Coord.) (2016). Educación rural: experiencias y propuestas de mejora. México: ColofónUniversidad Autónoma de Sinaloa, RIER.

Juárez, D. (2018). Politicas de cierre de escuelas rurales en América Latina. Ponencia presentada en el III Coloquio Iberoamericano de Educación Rural, Cusco, Perú.

Juárez, D. (Coord.) (2020). Politicas de cierre de escuelas rurales en Iberoamérica. Debates y experiencias. México: RIER-Editora Nómada.

Maalouf, A. (2019). El naufragio de las civilizaciones. México: Alianza Editorial.

MEJOREDU. (2020). 10 sugerencias para la educación durante la emergencia por COVID-19. México: Comisión Nacional para la Mejora Continua de la Educación.

MEN. (2015). Colombia territorio rural: apuesta por una política educativa para el campo. Colombia: Ministerio de Educación Nacional.

Mercado, R. (2002). Los saberes docentes como construcción social. La enseñanza centrada en los niños. México: Fondo de Cultura Económica.

Montero, C., Oliart, P., Ames, P., Cabrera, Z. y Uccelli, F. (2001). La escuela rural: modalidades y prioridades de intervención, (Documento de trabajo). pdf

Morales, N. (2012). La política de concentraciones escolares en el medio rural. Repercusiones desde su implantación hasta la actualidad. Ager, Revista de Estudios sobre Despoblación y Desarrollo Rural, (14), $145-187$.

Moscoso, J. A., Romero, L. y Tejero, M.C. (2018). La transposición didáctica interna de problemas aditivos en un preescolar multigrado. En Cano, A. e Ibarra, E. (Coords.), Vulnerabilidad, innovación y prácticas docentes en escuelas multigrado (117-139). México: RIER-UPES- Editora Nómada. 


\section{EST U D O S Y E S A Y S}

Naranjo, G. (2019). Aportes desde la inclusión educativa para la atención de población estudiantil con discapacidad y/o con "con NEE” en escuelas rurales. México. (Documento de trabajo).

Naranjo, G. (2019). Construcción situada de la enseñanza de contenidos científicos en un grupo multigrado. Ponencia en Congreso internacional Epistemologías del Sury Ruralidades Latinoamericanas. 9-11 de Octubre. Rionegro, Colombia. pdf

Parra, A. Mateus, J. y Mora, Z. (2018). Educación rural en Colombia: el país olvidado, antecedentes y perspectivas en el marco del posconflicto. Nodos y nudos, volumen 6 (45), 50-63.

Popoca, C. y Arteaga, P. (Coords.) (2019). Propuestas de Atención para la Educación Rural. México: RIER (Documento de trabajo).pdf

Rebolledo, V.y Torres, R.M. (Coords.) (2019). Estado del Arte de la educación rural en México (2004-2014). México: Universidad Iberoamericana, A.C. RIER.

RIER. (2016). Carta abierta al Secretario de Educación Pública. Recuperado de http://rededucacionrural. mx/eventos-y-noticias/avisos/comunicados/carta-abierta-al-secretario-de-educacion-publica/

Rivera, Á. y Zavala, M.A. (2019). Construcción de alternativas a problemáticas sentidas. Revisión del Modelo Educativo para el medio rural. Revista Latinoamericana de Educacióny Estudios InterculturalesRLEEI, volumen 3 (4) Octubre-Diciembre, 70-86.

Rodríguez, B.A. (Coord.) (2018). La enseñanza y aprendizaje de la lengua en escuelas rurales mexicanas. Diagnóstico e implementación en las aulas. México: UASLP-COLOFÓN-RIER.

Rosas, L. (2016). Maestros que trasforman su práctica educativa. Hacia una nueva escuela multigrado. México: Centro de Estudios Educativos.

Rosas, L. (2018). Los retos del aprendizaje en las escuelas rurales. México: Red Poniéndose al día.

Sánchez, N. (2018). Prácticas etnoeducativas en el departamento de Chocó: desafíos para una educación pertinente con la realidad y la vida. Nodos y nudos, volumen 6 (45), 96-103.

Santos, L. (2019). Educaciones y Ruralidades Latinoamericanas. Conferencia magistral. Congreso internacional Epistemologías del Sur y Ruralidades Latinoamericanas. 9-11 de Octubre. Rionegro, Colombia.

Schmelkes, S. y Águila, G. (Coords.) (2019). La educación multigrado en México. México: Instituto Nacional de Evaluación Educativa.

SEP. (2019). La Escuela es Nuestra. Manual del Comité Escolar de Administración Participativa. México: Secretaria de Educación Pública.

Sixto, C. (2014). Retos para la enseñanza en telesecundaria: los profesoresy sus posibilidades para resolverlos. Un estudio de escuelas de Michoacán. Tesis para obtener grado de Maestro en Ciencias en la Especialidad de Investigaciones Educativas. México: DIE-CINVESTAV-IPN

Solares, D. y Solares, A. (2018). Retos y alternativas para la enseñanza de las matemáticas en telesecundarias multigrado. Un estudio de caso. En Cano, A. e Ibarra, E. (Coords.), Vulnerabilidad, innovación y prácticas docentes en escuelas multigrado (pp. 89-116). México: RIER-UPES- Editora Nómada.

Torres, R.M. (2019). Profesión docente en educación básica rural. En Rebolledo, V. y Torres, R.M. (Coords.), Estado del Arte de la educación rural en México (2004-2014). (pp. 32-83). México: Universidad Iberoamericana, A.C. RIER. 


\section{EST U D O S Y E N A Y OS}

Villegas, M. (2017). Negociaciones y niveles de agencia en el postsecundario. El caso de las jóvenes de zonas rurales en Apurímac. En Ames, P. (Edit.). La diversidad en la escuela. Aproximaciones antropológicas a las experiencias educativas de los niños, niñas y jóvenes peruanos (pp. 109-131). Perú: Pontificia Universidad Católica del Perú- Instituto de Investigación para el Desarrollo.

Williamson, G. (2010). Todos en el mismo curso. Escuelas multigrado en la educación rural chilena: reflexiones desde algunas experiencias internacionales. e-Rural. (7),13-14.

Zamora, L. F. (2005). Huellas y búsquedas: semblanza de las maestras y maestros rurales colombianos. Bogotá: Fundación Universitaria Monserrate, Fundación Santa María.

Zamora, L. F. y Mendoza, A. (2018). La formación de educadores para el trabajo rural: el reto planteado por la escuela rural multigrado en Colombia. Nodos y nudos, 6 (45), 74-87.

Zegarra, A. (2017). Espacios que configuran dinámicas: repensando la Educación Intercultural Bilingüe en una comunidad shipibo. En Ames, P. (Edit.). La diversidad en la escuela. Aproximaciones antropológicas a las experiencias educativas de los niños, niñas y jóvenes peruanos (pp. 33-58). Perú: Pontificia Universidad Católica del Perú- Instituto de Investigación para el Desarrollo. 